\title{
Metacognitive Activities Inventory: Validade à Partir de uma Análise Fatorial Confirmatória
}

\author{
Raimundo Nonato Lima Filho ${ }^{1}$, Adriano Leal Bruni ${ }^{2}$
}

\begin{abstract}
Resumo: Este estudo tem o objetivo de traduzir e investigar evidências de validade do Inventário de Atividades Metacognitivas - MCAi, instrumento inédito na literatura em língua portuguesa. A tradução adotou a metodologia backtranslation e a validação foi através da Análise Fatorial Confirmatória pelo Modelo de Equações Estruturais. Foram coletadas 1058 respostas entre estudantes e profissionais de Administração na cidade Salvador, Bahia. O construto Atividade Metacognitiva, relacionado ao MCAi, confirmou que as dimensões Atividade Metacognitiva Planejamento, Atividade Metacognitiva Monitoramento e Atividade Metacognitiva Avaliação apresentaram medidas de confiabilidade composta e a validade discriminante corroborou que as correlações entre estes indicadores e suas respectivas dimensões são mais fortes que as correlações entre as dimensões; ou seja, o construto Atividade Metacognitiva reúne validade discriminante, o que permite validar o instrumento utilizado.
\end{abstract}

Palavras-chave: Metacognição; Tradução; Validação; Análise Fatorial; Validade Estatística.

\section{Metacognitive Activities Inventory: Validation From A Confirmatory Factor Analysis}

\begin{abstract}
This study aimed to translate and validate statistically the Metacognitive Activities Inventory - MCAI, new instrument in the literature in Portuguese. We collected 1058 responses from students and professionals in the city of Directors Salvador, Bahia. Translation adopted the methodology back translation and statistical validation was through Confirmatory Factor Analysis by Structural Equation Model. The construct Metacognitive Activity related to MCAI confirmed that the dimensions Metacognitive Activity Planning, Metacognitive Activity Monitoring and Metacognitive Activity Assessment presented measures of composite reliability and the discriminant validity confirmed that the correlations between these indicators and their dimensions are stronger than the correlations between dimensions, ie, the construct Metacognitive Activity meets discriminant validity, which allows to validate the instrument used.
\end{abstract}

Keywords: Metacognition; Translation; Validation; Factor Analysis; Statistical Validity.

\footnotetext{
${ }^{1}$ Doutor em Controladoria e Contabilidade pela Universidade de São Paulo (USP) e Doutor em Administração pela Universidade Federal da Bahia (UFBA), possui Pós-Doutorado pela Universidade Federal da Paraíba (UFPB). É Professor da Autarquia Educacional do Vale do São Francisco (AEVSF). Pesquisador dos grupos de pesquisa: Grupo de Estudos de Tecnologia da Educação na Contabilidade (USP), Contabilidade e Controladoria (UFBA), Laboratório de Pesquisa em Contabilidade de Gestão (UFBA), Núcleo de Estudos em Contabilidade Financeira e Finanças Empresariais (UFBA) e Grupo de Pesquisa em Finanças e Contabilidade (UFPB). Contato: rnlfilho@gmail.com;

${ }^{2}$ Professor Titular da Faculdade de Ciências Contábeis da Universidade Federal da Bahia, Doutor (2002) e Mestre (1998) em Administração pela Universidade de São Paulo. Contato: albruni@ufba.br.
} 


\section{Introdução}

Flavell (1976) elaborou o termo metacognição e a delineou como "o conhecimento sobre fenômenos cognitivos" (YORE; TREAGUST, 2006). Inicialmente, essa definição pode parecer simples, entretanto, Thomas, Anderson e Nashon (2008) argumenta que apesar da quantidade considerável de pesquisas sobre metacognição e seu impacto na compreensão humana, não há um consenso em sua definição. Diferentes significados podem ser encontrados na literatura atual (KAUFFMAN; GE; XIE; CHEN, 2008; SCHUNK, 2008; THOMAS et al., 2008), todavia, a definição mais empregada é o conhecimento e regulação do próprio sistema cognitivo (BROWN, 1987).

Pode ser mais facilmente entendida como consciência de como se aprende; consciência de quando se faz; o conhecimento de como usar as informações disponíveis para alcançar um objetivo; capacidade de julgar as demandas cognitivas de uma determinada tarefa; conhecimento das estratégias a serem utilizadas (GOURGEY, 2001).

A metacognição ocorre justamente quando os indivíduos planejam, monitoram e avaliam seu próprio pensamento em um ambiente de aprendizagem ou de resolução de problemas.

Modelos teóricos, bem como elementos de pesquisa, sugerem a existência de dois elementos principais da metacognição: conhecimento metacognitivo e habilidade metacognitiva, que também são referidos como o conhecimento da cognição e regulação da cognição, respectivamente. Conhecimento da cognição descreve a consciência de um indivíduo em três níveis diferentes: declarativo (saber sobre as coisas), procedimental (saber sobre como fazer as coisas), e condicional (saber por que e quando fazer as coisas). Regulação da cognição refere-se às atividades e ações realizadas por indivíduos para controlar a sua própria cognição (SCHRAW, 2001).

Davidson, Deuser e Stenberg (1994) identificaram um número de diferentes atividades de regulação agrupadas em três categorias: planejamento, acompanhamento e avaliação. Essas competências reguladoras orientam o processo de resolução de problemas e seu refinamento melhora a eficiência na resolução de problemas e outras tarefas. 
Assim como a variável metacognição, em diversos elementos das ciências humanas, sociais ou da saúde, é impossível analisar, tampouco medir algumas variáveis, conhecidas como variáveis latentes. A manifestação destas variáveis latentes é uma das maneiras encontradas pelos psicometristas para observá-las. A calibração destes instrumentos não é uma tarefa fácil, uma vez que não existe um modelo de calibração universal e consensual. É necessário, portanto, recorrer a técnicas estatísticas que possibilitam cotejar a confiabilidade e validade dos dados coletados com os instrumentos de medida para variáveis latentes.

Deste modo, é de grande relevância e contribuição para os estudos em Psicologia, não só o desenvolvimento de instrumentos de medida, como também a tradução e validação de instrumentos já consolidados na literatura, disponibilizando-os para o contexto nacional.

Em consulta ao Sistema de Avaliação de Testes Psicológicos do Conselho Federal da Psicologia (SATEPSI), em abril de 2013, foi identificado que o Metacognitive Activities Inventory (MCAi) não estava na lista homologada de testes psicológicos aprovados, o que motivou o objetivo desta pesquisa, que é sua tradução e investigação de evidências de validade.

O Metacognitive Activities Inventory (MCAi) - Tabela 1 - é um instrumento psicométrico desenvolvido por Cooper, Sandí-Ureña e Stevens (2008) caracterizando-se como um inventário de autorrelato (o próprio respondente deve preencher). O MCAi foi elaborado para ser administrado e analisado de maneira rápida e fácil a qualquer momento em população de estudantes em nível de graduação.

Tabela 1 - Metacognitive Activities Inventory-Versão Brasileira

1. Eu leio a declaração de um problema cuidadosamente para compreender e determinar qual é a meta.

2. Quando eu resolvo problemas, eu tento aprender mais sobre os conceitos para que eu possa aplicar posteriormente esse conhecimento.

3. Eu separo as informações da declaração e determino o que é relevante.

4. Uma vez que o resultado é obtido, eu verifico se está de acordo com que eu esperava.

5. Eu tento relacionar problemas desconhecidos com situações anteriores ou problemas resolvidos.

6. Eu tento determinar a forma em que a resposta vai ser expressa.

7. Se um problema envolve vários cálculos, eu faço esses cálculos separados e verifico os resultados intermediários.

8. Eu claramente identifico o objetivo de um problema (a variável desconhecida para resolver ou o conceito a ser definido) antes de tentar uma solução.

9. Eu considero que a informação necessária pode não ser apresentada na declaração do problema. 
10. Eu tento verificar tudo novamente: a minha compreensão do problema, os cálculos, as unidades, etc.

11. Eu uso organizadores gráficos (diagramas, fluxogramas) para melhor compreender um problema.

12. Eu experimento momentos de criatividade na resolução de problemas.

13. Eu anoto coisas que eu sei que poderiam me ajudar a resolver um problema, antes de tentar uma solução.

14. Procuro relações importantes entre as quantidades, fatores ou conceitos envolvidos antes de tentar uma solução.

15. Procuro ter certeza de que minha solução realmente responde a pergunta.

16. Eu planejo como resolver um problema antes de realmente começar a resolvê-lo (mesmo que seja um breve plano mental).

17. Reflito sobre coisas que eu sei que são relevantes para um problema.

18. Eu analiso os passos do meu plano e a adequação de cada etapa.

19. Eu tento fragmentar o problema para encontrar o ponto de partida.

20. Gasto pouco tempo em problemas para os quais eu não tenho um conjunto de regras de solução ou que não fui ensinado antes.

21. Quando eu resolvo problemas, penso em conceitos antes de tentar uma solução.

22. Uma vez que eu sei como resolver um tipo de problema, eu não gasto muito tempo na compreensão dos conceitos envolvidos.

23. Eu não verifico se a resposta faz sentido.

24. Se eu não sei exatamente como resolver um problema, eu imediatamente tento adivinhar a resposta.

25. Eu começo a resolver problemas sem ter que ler todos os detalhes da declaração.

26. Passo pouco tempo em problemas que eu não tenho certeza se posso resolver.

27. Se tentei várias vezes resolver um problema, e não consegui, procuro alguém que consiga resolver e memorizo o procedimento.

Fonte: Sandí-Ureña (2008) tradução Lima Filho (2013)

Conforme metodologia backtranslation de Pietro (1992), os 27 itens do MCAi foram traduzidos do inglês para o português por três tradutores bilíngues que trabalharam de forma independente, gerando, portanto, três versões em português. Essas versões foram traduzidas para o inglês por outros três tradutores bilíngues, também trabalhando de forma independente, e, finalmente, um último tradutor bilíngue, analisando todas as traduções, realizou os ajustes necessários para se obter uma única versão apropriada do instrumento em português, garantindo, assim, a equivalência de sentido.

Sandí-Ureña (2008) discute a importância da metacognição na aprendizagem e na resolução de problemas. $\mathrm{O}$ autor afirma que apesar do relevante número de publicações na área, há pouco interesse no desenvolvimento de instrumentos que mensurem a metacognição e constructos correlatos. Um dos motivos para esse desinteresse pode estar nas dificuldades 
intrínsecas da caracterização dos padrões individuais de pensamento e desenvolvimento de estratégia (SPERLING; HOWARD; STALEY, 2002).

Identificando essa lacuna na literatura, Cooper, Sandí-Ureña e Stevens (2008) desenvolveram o MCAi com o objetivo de fornecer aos educadores uma ferramenta que permita uma compreensão mais profunda de como os estudantes percebem suas habilidades para resolver problemas e atividades.

$\mathrm{O}$ instrumento foi validado utilizando critérios de confiabilidade e validade. A confiabilidade foi analisada através do teste de alfa de Cronbach (nível de 0,91) e a validade foi examinada por meio da validade do constructo e do conteúdo. O MCAi apresentou um coeficiente de Correlação de Pearson em 0,53, com grau de significância a 0,01\%; a análise da variância das médias apresentou ser estatisticamente diferente $(0,015)$, o que permitiu derivar evidências de validade ao instrumento.

Modelos teóricos sugerem a existência de dois componentes principais da metacognição: o conhecimento e habilidades metacognitivas e as estratégias da regulação (as habilidades metacognitivas de planejamento, monitoramento e avaliação são analisadas por meio de itens no MCAi). A partir dessa discussão, apresenta-se na Figura 1 o enfoque do MCAi.

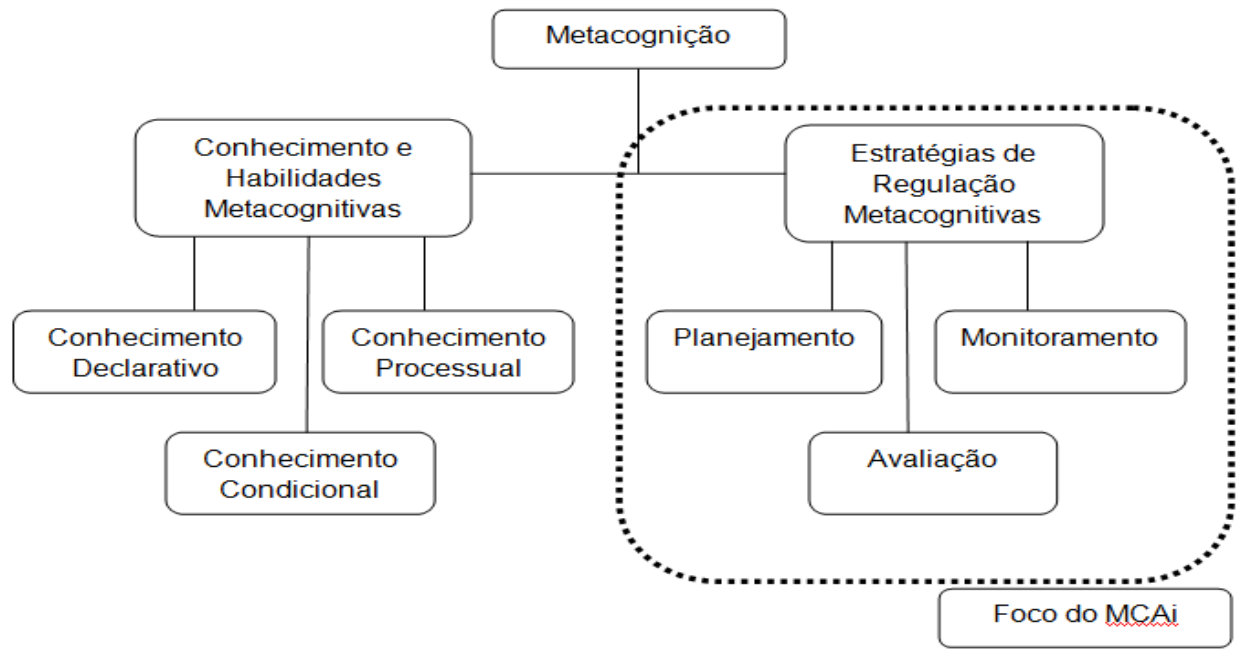

Figura 1. Componentes do Modelo Teórico para a Metacognição Fonte: Adaptado - Cooper, Sandí-Ureña e Stevens (2008). 
A partir da Figura 1, percebe-se que o enfoque do MCAi está somente na perspectiva das Estratégias de Regulação Metacognitivas. No entanto, as tentativas de classificar os itens como pertencentes a apenas uma das subcategorias principais de planejamento, acompanhamento e avaliação não foram bem sucedidas. Isso já era esperado pelos pesquisadores, uma vez que há uma elevada interdependência entre as competências metacognitivas, o que implica que alguns itens se enquadrem em mais de uma subcategoria.

Cooper et al. (2008) utilizaram a técnica painel de especialistas para a primeira etapa da construção do instrumento. Nessa etapa foram consultados quatro professores doutores e quatro alunos scricto sensu. Cada especialista consultado listou dez habilidades ou atividades que julgavam estar relacionados à atividades metacognitivas. As contribuições foram listadas de acordo com o método de Thorndike (2005), sendo que uma parte dos itens precisou receber uma codificação inversa, com a finalidade de evitar os efeitos da aquiescência (tendência dos respondentes em concordar com as declarações apresentadas).

Em seguida, uma lista preliminar com 53 itens foi analisada pelos desenvolvedores e por um especialista em psicologia educacional. Um dos principais critérios empregados nessa fase da elaboração do instrumento foi assegurar que os outputs estivessem ligados às Estratégias de Regulação Metacognitivas.

Após um pré-teste aplicado em 151 estudantes de graduação em Química, os desenvolvedores do MCAi realizaram uma seleção final dos itens a partir de dois critérios: a correlação individual dos itens com o escore final e o efeito exclusão sobre o alfa de Cronbach, onde os itens que pouco ou nada contribuíam para este índice foram excluídos. Ao final, os pesquisadores chegaram ao instrumento definitivo com 27 outputs, conforme a Tabela 1.

O instrumento definitivo foi aplicado, em um período máximo de 15 minutos, em uma amostra de 310 estudantes de graduação, sendo que 280 questionários foram preenchidos corretamente. $\mathrm{O}$ instrumento foi validado pelos autores, através de teste $\mathrm{t}$, coeficiente de correlação de Pearson, alfa de Cronbach e análise de variância.

Os desenvolvedores afirmaram que o MCAi foi idealizado para medir três fatores. O primeiro fator (12 itens) - Atividade Metacognitiva Planejamento (AMEP) - é um fator que analisa as medidas tomadas antes da resolução de problemas. Inclui o estabelecimento de metas e recursos alocados. O segundo fator (10 itens) - Atividade Metacognitiva 
Id on Line Revista Multidisciplinar e de Psicoloqia

Id on Line Multidisciplinary and Psycology Journal

Monitoramento (AMEM) - é um fator que avalia as decisões durante a resolução de problemas; e o terceiro fator (5 itens) - Atividade Metacognitiva Avaliação (AMEA) - é um fator que envolve as ações tomadas após a resolução de problemas. A pontuação resultante é uma porcentagem do máximo de pontos possíveis, em que quanto maior a pontuação, maior é o nível de atividade metacognitiva do indivíduo, conforme Tabela2.

Tabela2 - Atividades Metacognitivas e Questões por Categoria

\begin{tabular}{c|c}
\hline CATEGORIA: PLANEJAMENTO (AMEP) & Questões \\
\hline Determinação do enfoque do problema & 11 \\
\hline Acessar informações prévias & $6,8,14$ e 25 \\
\hline Alocar recursos & $13,24,26$ e 27 \\
\hline Orçar o tempo & 7,12 e 16 \\
\hline Autoteste & $2,17,18,19,20$ e 21 \\
\hline CATEGORIA: MONITORAMENTO (AMEM) & $3,5,21$ e 22 \\
\hline CATEGORIA: AVALIAÇÃO (AMEA) & 9 e 10 \\
\hline Avaliar produtos & 4,15 e 23 \\
\hline Reavaliar objetivos e conclusões & \\
\hline
\end{tabular}

Fonte: Jordan (2011)

Além da pesquisa original, o MCAi já foi validado em algumas pesquisas internacionais (DELVECCHIO, 2011; JORDAN, 2011; BRUCK, 2011; TÜYSÜZ, 2009), o que corrobora a sua utilização nesta pesquisa, além de que sua tradução e validação contribuirá para próximas pesquisas em língua portuguesa interessadas neste instrumento psicométrico.

\section{Método}

Utilizou-se do critério de acessibilidade para compor a amostra e adota a sugestão de Hair et al. (2005), que indica, como regra geral, que a base de dados de uma pesquisa envolva 
no mínimo cinco vezes mais observações do que o número de variáveis que compõe o número de dados. Como o instrumento de coleta de dados apresenta 27 questões, esta pesquisa precisou coletar uma amostra com no mínimo 135 respondentes.

O estudo para validação do MCAi foi efetivado por meio da análise de uma amostra formada por estudantes e graduados em Administração de Salvador (BA). A amostra foi composta por: (a) alunos de IES públicas e privadas e (b) graduados registrados no Conselho Regional de Administração do Estado da Bahia (CRA-Ba).

A coleta de dados foi realizada a partir da aplicação de questionário eletrônico via Google Docs, disponibilizado pelo CRA-Ba e pelas coordenações de curso das IES envolvidas na pesquisa ( 3 públicas e 6 privadas). A amostra foi composta por 1058 respondentes, sendo 851 graduados e 207 estudantes.

De acordo com o objetivo deste estudo, o tratamento dos dados foi desenvolvido em duas fases. Na primeira, apresenta uma análise descritiva com o objetivo de caracterizar os respondentes da amostra e estudar a distribuição dos dados. Na segunda fase, foi aplicada a Análise Fatorial Confirmatória (AFC) pelo Modelo de Equações Estruturais propostos por Venkatraman e Grant (1986), para testar a validade de construto que envolve a análise de confiabilidade e consistência interna dos indicadores; a validade convergente e a validade discriminante da dimensão do construto: "Atividade Metacognitiva" (MCAi).

$\mathrm{O}$ modelo teórico desta pesquisa almejou expor fatores relacionados à Atividade Metacognitiva, para tanto, foi empregado o instrumento de pesquisa anteriormente citado, composto por 27 questões, cujas respostas, a priori, esperam ser explicadas por três fatores: (a) Atividade Metacognitiva Planejamento (AMEP); (b) Atividade Metacognitiva Monitoramento (AMEM); e (c) Atividade Metacognitiva Avaliação (AMEA), conforme Tabela2. 


\section{Resultados}

\section{Fase 1 - Análise descritiva dos dados}

A análise descritiva dos dados coletados e processados com o software Statistical Package for Social Sciences (SPSS) revelou que a amostra foi composta por 1058 respondentes, dos quais 55,7\% (589) do gênero masculino e 44,3\% (469) do gênero feminino, com idade média de 35,9 anos e desvio-padrão 11,24 anos. Desses respondentes 80,4\% (851) são graduados, $19,6 \%$ (207) estudantes.

O teste de normalidade multivariada dos dados resultou na estatística de curtose multivariada PK de Mardia Normalizada ( $\mathrm{PK}=105,37 ; p=0,001)$. Como já era esperado, a distribuição não apresenta normalidade multivariada uma vez que os dados coletados derivam de escalas ordinais (conforme instrumento de pesquisa).

Tabela 3-Composição da amostra por idade

\begin{tabular}{r|c|c}
\hline & Frequência & Proporção \\
\hline Entre 18 e 28 anos & 291 & 27,5 \\
Entre 29 e 38 anos & 398 & 37,6 \\
Entre 39 e 48 anos & 199 & 18,8 \\
Entre 49 e 58 anos & 124 & 11,7 \\
Acima de 59 anos & 46 & 4,3 \\
Fonte: elaboração própria, 2016.
\end{tabular}

Em relação à distribuição da idade dos respondentes por faixa, 65,1\% dos respondentes apresentaram idade inferior a 38 anos. Os respondentes mais jovens desta pesquisa possuem 18 anos ( 7 indivíduos) e os mais maduros possuem 72 anos ( 2 indivíduos). O teste de normalidade multivariada dos dados resultou na estatística de curtose multivariada PK de Mardia Normalizada $(\mathrm{PK}=81,01 ; p=0,001)$, portanto a distribuição não apresenta normalidade multivariada. 
Quando considerada segundo o gênero, a média de idade das mulheres (Média=34,14; Desvio-padrão=0,458) é menor que a dos homens (Média=37,41; Desvio Padrão=0,494) e as distribuições de frequência são assimetricamente positivas para ambos os gêneros, conforme ilustra o histograma da Figura 2.

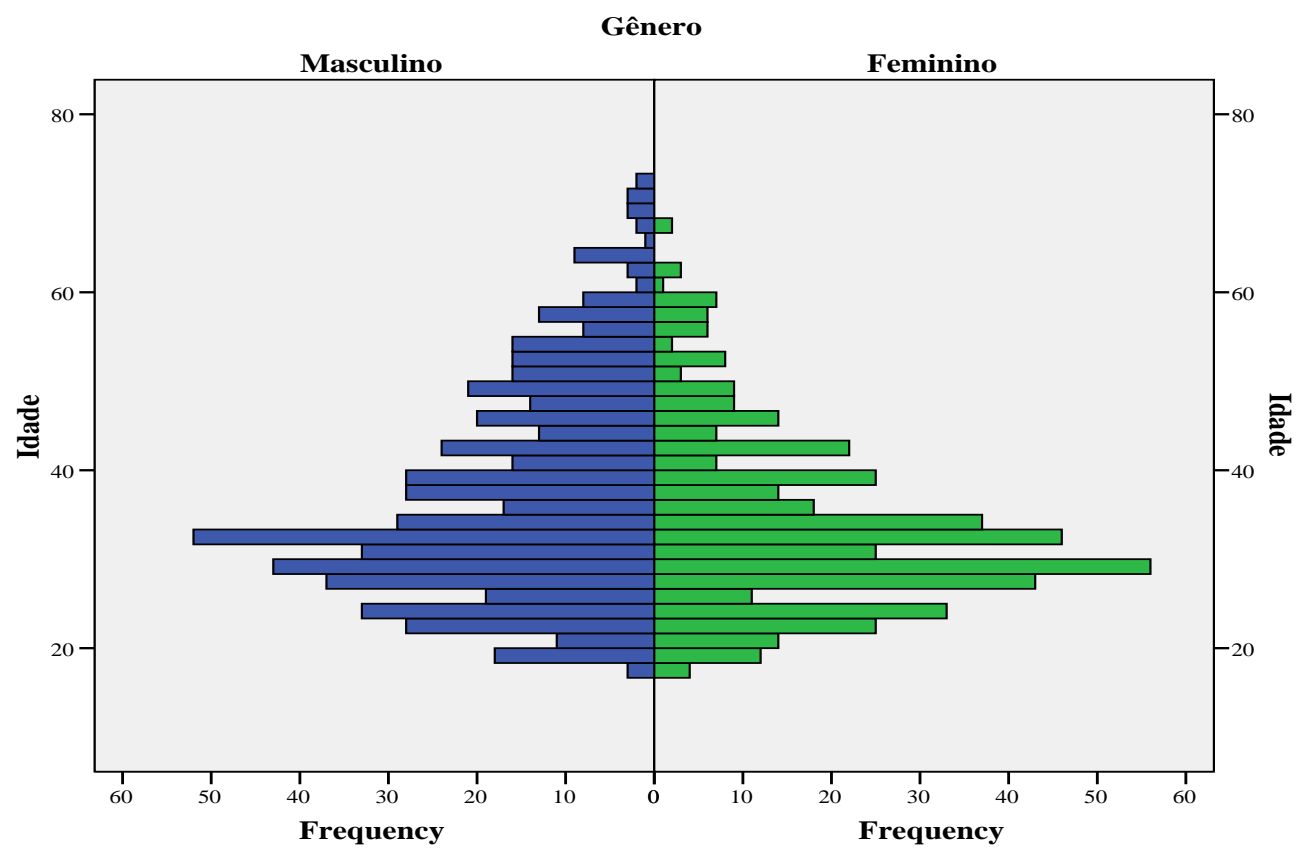

Figura 2 - Distribuição da idade por gênero Fonte: elaboração própria, 2016.

\section{Fase 2 - Validação do instrumento}

A validade do construto "Atividades Metacognitivas" (MCAi) foi avaliada a partir do processamento do modelo reflexivo com três dimensões: a) Atividade Metacognitiva Planejamento (AMEP); b) Atividade Metacognitiva Monitoramento (AMEM); e c) Atividade Metacognitiva Avaliação (AMEA) estimadas a partir dos 27 indicadores observáveis, pelo algoritmo Path Weighting Scheme com reposição de missing values pelo valor médio e normalização (Média=0; Variância=1). No entanto, o processamento resultou na retirada dos 
Id on Line Revista Multidisciplinar e de Psicoloqia

Id on Line Multidisciplinary and Psycology Journal

indicadores apresentados na Tabela 4 por apresentar baixa carga fatorial com os demais indicadores das dimensões com que se relacionavam.

Tabela 4 - Indicadores excluídos do instrumento

\begin{tabular}{cccccc}
\hline $\mathrm{P}$ & Carga & $\mathrm{P}$ & Carga & $\mathrm{P}$ & Carga \\
\hline 2 & 0,204 & 13 & 0,322 & 22 & 0,144 \\
6 & 0,014 & 14 & 0,123 & 24 & 0,103 \\
7 & 0,022 & 16 & 0,176 & 25 & 0,077 \\
8 & 0,115 & 17 & 0,219 & 26 & 0,159 \\
9 & 0,345 & 18 & 0,033 & 27 & 0,101 \\
10 & 0,099 & 19 & 0,076 & & \\
12 & 0,278 & 20 & 0,087 & & \\
\hline
\end{tabular}

Fonte: Elaboração própria, 2016.

As cargas fatoriais resultantes do novo processamento do modelo desse indicador, ilustradas na Figura 3 indicam que o construto reúne, minimamente, validade convergente.

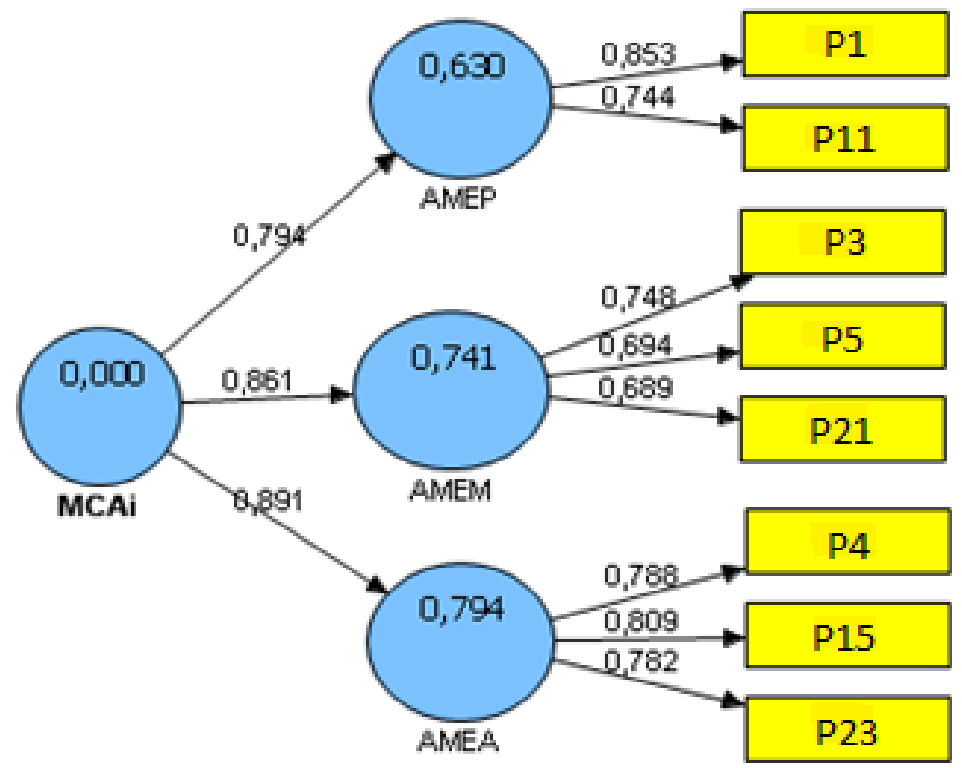

Figura 3 - Construto Atividade Metacognitiva - MCAi Fonte: elaboração própria, 2016.

A consistência interna dos indicadores foi avaliada pelo índice de confiabilidade composta, que segundo Hair et al. (2005) tem sido frequentemente utilizado nos modelos confirmatórios com MEE-PLS e calculado por meio da equação: 


$$
\text { Conf }=\frac{\left(\Sigma \lambda_{i}\right)^{2}}{\left(\Sigma \lambda_{i}\right)^{2}+\Sigma \operatorname{var}\left(\varepsilon_{i}\right)} \quad \operatorname{var}\left(\varepsilon_{i}\right)=1-\lambda_{i}^{2} \quad \text { Equação } 1
$$

As medidas de confiabilidade composta das dimensões AMEP, AMEM e AMEA foram respectivamente iguais a $(0,7801 ; 0,8608$ e 0,8911$)$, ou seja, ficaram acima do patamar de 0,70 indicado na literatura de Modelagem de Equações Estruturais, conforme Chin (1998), Fornell e Larcker (1981) e Hair et al. (2005); dessa forma, podem ser consideradas consistentes e confiáveis para mensurar o construto "Atividades Metacognitivas" (MCAi).

As correlações entre as dimensões do construto MCAi e suas respectivas Raízes da AVE foram organizadas na Tabela 5 para avaliação da validade discriminante.

Tabela 5 - Matriz de correlações construto MCAi

\begin{tabular}{ccccc}
\hline & AMEA & AMEM & AMEP & MCAi \\
\hline AMEA & $\mathbf{0 , 7 9 3 1 7 5}^{\mathbf{a}}$ & & & \\
AMEM & 0,630506 & $\mathbf{0 , 7 1 0 8 3 6 8}^{\mathbf{a}}$ & & \\
AMEP & 0,558402 & 0,572112 & $\mathbf{0 , 8 0 0 3 8 2 4}{ }^{\mathbf{a}}$ & \\
MCAi & 0,891133 & 0,860776 & 0,793852 & $\mathbf{0 , 8 4 9 5 2 9 3}^{\text {a }}$ \\
\hline $\begin{array}{l}\text { Fonte: elaboração própria, 2016. } \\
\text { a.Raiz da AVE }\end{array}$
\end{tabular}

Os dados da Tabela 5 mostram que Raiz da AVE de cada dimensão do construto MCAi excede as correlações entre as dimensões desse construto evidenciando que o relacionamento entre os indicadores e suas respectivas dimensões é mais forte que as correlações entre as dimensões. Consequentemente, o construto MCAi também reúne validade discriminante.

Observa-se também a existência de fortes correlações entre dimensões AMEP, AMEM e AMEA e o construto MCAi (0,7938; 0,8608 e 0,8911); logo, esse construto explica a variabilidade ( $\mathrm{R}^{2}$ da matriz de correlação) dessas dimensões em 60,85\%; 74,10\% e 79,40\%. Portanto, o MCAi foi validado estatisticamente, considerando a exclusão dos fatores não significativos, e suas dimensões podem ser utilizadas para estimar reflexivamente o construto "Atividade Metacognitiva" (MCAi). 
O instrumento validado por esta pesquisa é demonstrado no Tabela 6 .

Tabela 6 -Metacognitive Activities Inventory - Traduzido e Validado

1. Eu leio a declaração de um problema cuidadosamente para compreender e determinar qual é a meta.

2. Eu separo as informações da declaração e determino o que é relevante.

3. Uma vez que o resultado é obtido, eu verifico se está de acordo com que eu esperava

4. Eu tento relacionar problemas desconhecidos com situações anteriores ou problemas resolvidos.

5. Eu uso organizadores gráficos (diagramas, fluxogramas) para melhor compreender um problema.

6. Procuro ter certeza de que minha solução realmente responde a pergunta.

7. Quando eu resolvo problemas, penso em conceitos antes de tentar uma solução.

8. Eu não verifico se a resposta faz sentido.

Fonte: Sandí-Ureña (2008)

No instrumento validado, ficaram vinculadas ao construto AMEP as questões 1 e 5, ao constructo AMEM as questões 2, 4 e 7, e ao construto AMEA as questões 3, 6 e 8.

\section{Conclusões}

Esta pesquisa teve o objetivo traduzir e validar o Metacognitive Activities Inventory (MCAi) desenvolvido por Cooper et al. (2008). A tradução adotou a metodologia de Pietro (1992) e a validação estatística foi por meio da Análise Fatorial Confirmatória pelo Modelo de Equações Estruturais.

Dos 27outputs do instrumento original, este estudo encontrou que somente 8 (oito) outputs são significativos, pelo menos na conjuntura da análise desta pesquisa. Contudo, os outputs significativos conseguiram explicar o construto "Atividade Metacognitiva" e confirmaram que as dimensões Atividade Metacognitiva Planejamento (AMEP), Atividade Metacognitiva Monitoramento (AMEM) e Atividade Metacognitiva Avaliação (AMEA), apresentam medidas de confiabilidade composta. Da mesma forma, a validade discriminante corroborou que as correlações entre estes indicadores e suas respectivas dimensões são mais fortes que as correlações entre as dimensões, ou seja, o construto "Atividade Metacognitiva" reúne validade discriminante, o que permite atingir o objetivo deste estudo.

A contribuição desta pesquisa, além de sua tradução, foi de demonstrar que o instrumento validado consegue confirmar que o MCAi é responsável por $60,85 \% ; 74,10 \%$ e 
79,40\% da variabilidade dos construtos AMEP, AMEM e AMEA, apesar da relevante exclusão dos outputs do instrumento original.

Podem-se destacar algumas limitações importantes deste estudo, as quais podem ser vistas como oportunidades para pesquisas futuras. A amostra da pesquisa foi restrita a cidade de Salvador e em estudantes e profissionais de Administração. Estudos futuros poderiam aplicar o instrumento em outros contextos regionais e/ou em outros tipos de respondentes para analisar o comportamento dos construtos no contexto de sua pesquisa e corroborar ou refutar os achados deste estudo.

\section{Referências}

BROWN, A. L. Knowing when, where, and how to remember: A problem of metacognition. Advances in Instructional Psychology, v. 1, p. 77-165, 1987.

BRUCK, A. D. Faculty Perspectives of the Undergraduate Laboratory: A Survey of Faculty Goals for the Laboratory and Comparative Analysis of Responses Using Statistical Techniques. Tese (Doutorado em Filosofia), 317p., Purdue University Policies, EUA, 2011.

CHIN, W. W. The partial least squares approach for structural equation modeling. In George A. Marcoulides (Ed.), Modern Methods for Business Research, Lawrence Erlbaum Associates, 1998.

COOPER, S. S. Metacognition in the adult learner. Tese de Doutorado. Universidade Weber State, 2004.

COOPER, M. M.; SANDÍ-UREÑA, S.; STEVENS, S. Reliable multi method assessment of metacognition use in chemistry problem solving. Chemistry Education Research and Practice, v. 9, p. 18-24. DOI: 10.1039/B801287N, 2008.

DAVIDSON, E.; DEUSER I.; STENBERG, R. Metacognition: Knowing about knowing. METCALFE \& SHIMAMURA (Orgs.), Metacognition. Bradford Book The MIT Press Cambridge: Massachusetts, 1994.

DELVECCHIO, F. Student' use of Metacognitive Skills while Problem Solving in High School Chemistry. Dissertação de Mestrado, 161 f., Universidade de Queen’s, 2011.

FLAVELL, J. H. Metacognitive aspects of problem solving. In L. B. Resnick (Ed.), The nature of intelligence (pp.231-236). Hillsdale, NJ: Erlbaum, 1976. 
FORNELL, C.; LARCKER, D. Evaluation Structural Equation Models with Unobservable Variables and Measurement Error. Journal of Marketing Research, v. 18, p. 39-50. DOI: $10.2307 / 3151312,1981$.

GOURGEY, A. F. Developing students' metacognitive knowledge and skills. In H.J. HARTMAN (Ed.), Metacognition in learning and instruction: theory, research and practice. Kluwer Academic Publishers: The Netherlands, 683T.17-32, 2001.

HAIR, J. F.; ANDERSON, R. E.; TATHAM, R. L.; BLACK, W. C. Multivariate Data Analysis. 5. Ed. New Jersey: Prentice Hall, 2005.

JORDAN, J. R. Teacher Practices and High School Chemistry Students' Metacognitive Skillfulness. Tese de Doutorado, 186 p., Universidade de Clemson, 2011.

KAUFFMAN, D. F.; GE, X.; XIE, K.; CHEN, C. Prompting in web-based environments: Supporting self-monitoring and problem solving skills in college students. Journal of Educational Computing Research, 38(2), p. 115-137. DOI: 10.2190/EC.38.2.a, 2008.

PIETRO, A. J. A method for translation of instruments to other languages. Adults Education Quartely, v. 43, n. 1, p. 1-14. DOI: 10.1177/0741713692043001001. 1992.

SANDÍ-UREÑA, G. S. Design and Validation of a Multimethod Assessment of Metacognition and Study of the Effectiveness of Metacognitive Interventions. Tese de Doutorado (Doutorado em Química), Universidade de Clemson, 175 f, 2008.

SCHRAW, G. Promoting general metacognitive awareness. Metacognition in learning and instruction: theory, research and practice. Nova York, Kluwer Academic Publishers, 2001.

SCHUNK, D. H. Commentary on self-regulation in school contexts. Learning and Instruction, 15, 173-177. DOI: 10.1016/j.learninstruc.2005.04.013, 2008.

SPERLING, R. A.; HOWARD, B. C.; STALEY, R. Metacognition and self-regulated learning constructs. Educational Research and Evaluation, v. 10, p. 117-139. DOI: 10.1076/edre.10.2.117.27905, 2004.

THOMAS, G.; ANDERSON, D.; NASHON, S. Development of an instrument designed to investigate elements of science students' metacognition, self-efficacy and learning processes: The SEMLI-S. International Journal of Science Education, v. 30, n. 13, p. 1701-1724, 2008.

THORNDIKE, R .M. Measurement and evaluation in psychology and education. New Jersey: Pearson Prentice Hall, 2005. 
TÜYSÜZ, C. Effect of the computer based game on pre-service teachers' achievement, attitudes, metacognition and motivation in chemistry. Scientific Research and Essay, v. 4, n. 8, p. 780-790, 2009.

VENKATRAMAN, N.; GRANT, J. H. Construct Measurement in organizational Strategy Research: A Critique and Proposal. Academy of Management Review, v. 11, n. 1, p. 71-87, 1986.

YORE, L. D.; TREAGUST, D. F. Current realities and future possibilities: Language and science literacy-empowering research and informing instruction. International Journal of Science Education, v. 28, n. 2-3, p. 291-314. DOI: 10.1080/09500690500336973, 2006.

\section{Como citar este artigo (Formato ABNT):}

LIMA FILHO, Raimundo N.; BRUNI, Adriano L. Metacognitive Activities Inventory: Validade à Partir de uma Análise Fatorial Confirmatória. Id on Line Revista Multidisciplinar e de Psicologia, 2017, vol.11, n.37, p. 669-684. ISSN: 1981-1179.

Recebido: $25 / 09 / 2017$

Aceito: $27 / 09 / 2017$ 